\title{
LA PERSPECTIVA DE GÉNERO EN LA FILOSOFÍA
}

\author{
SONIA REVERTER BAÑÓN ${ }^{1}$
}

Universitat Jaume I

\section{LA IGUALDAD COMO CONCEPTO DE VINDICACIÓN FILOSÓFICA²}

La filosofía occidental cuenta con una larga tradición patriarcal que en líneas generales se mantiene bastante homogénea hasta llegar a la Ilustración. La intensa labor histórica de recuperación de voces que muestren la plena capacidad de pensamiento de las mujeres que se ha llevado a cabo en el siglo XX, nos ha convencido de que podemos mantener que la filosofía, pese a presentarse tradicionalmente como "una cosa de hombres», nunca ha sido ajena a las mujeres.

Así, si rastreamos (puesto que se convierte casi en una labor detectivesca) podemos encontrarnos desde los mismos inicios en la época griega con voces de mujeres que, contra los marcos culturales de dominación, hacían filosofía, demostrando así con ello que la denigración que la filosofía ortodoxa ha mostrado a lo largo de la historia por las mujeres era rebatible en sus mismos fundamentos.

La tarea de rastreo ha ido combinada con una labor de verdadera construcción filosófica de todo un cuerpo teórico múltiple y variado denominado «teoría feminista». Actualmente este cuerpo teórico es parte importante en multitud de departamentos e institutos universitarios, dejando claro que la dimensión de la perspectiva de género no es ya una cuestión «de mujeres», sino que el esfuerzo de reconceptualización va más allá del interés político que las mujeres podamos tener.

Podemos decir que la producción feminista en filosofía comenzó con la Ilustración, es decir, cuando las estructuras políticas, sociales y culturales se empezaron a fundamentar en la racionalidad científica, la razón, la igualdad y

1. Departamento de Filosofía, Sociología, Comunicación Audiovisual y Publicidad, Universitat Jaume I Castellón. Reverter@fis.uji.es. El presente trabajo es fruto parcial de la investigación llevada a cabo por el grupo del proyecto financiado por Fundación Bancaixa-Universitat Jaume I, P1. 1B 2002-27.

2. Todas las traducciones que aparezcan en el texto de los originales en lengua inglesa son mías. 
la justicia social. En este período se empezó, como es bien sabido, la deslegitimación de las fuentes de autoridad tradicionales, es decir, las jerarquías políticas absolutistas y religiosas. Se gestaron los conceptos de sociedad civil, libertad civil, derechos de la ciudadanía, contrato social, y autonomía racional, que permitieron proyectos como las revoluciones americana y francesa. El feminismo, sin ser en realidad parte del proyecto ilustrado, tuvo un apropiado caldo de cultivo en estas ideas, que son en líneas básicas el núcleo de las reivindicaciones de las luchas de las mujeres aún hoy. El modelo ilustrado, teórica y brillantemente orquestado sobre el concepto de igualdad, se contradecía en la práctica al reducir la «universalidad» a un sujeto varón, blanco, y de una determinada clase social. La idea de igualdad, con su enorme potencia, se había convertido en un grito que los grupos de excluidos hicieron suyo. Las mujeres fue uno de esos grupos que se apropió de esta idea, gracias a lo cual el feminismo se acaba convirtiendo en lo que audazmente Amelia Valcárcel ${ }^{3}$ ha llamado «un hijo no querido de la Ilustración", y Celia Amorós ${ }^{4}$ de manera incisiva ha denominado "radicalización de la Ilustración», y por tanto "test de coherencia» de la misma. Los autores ilustrados más representativos, desde Kant a Rousseau, serán incapaces de estar a la altura de las exigencias del concepto de igualdad que vitoreaban. Como bien afirma Celia Amorós ${ }^{5}$ inspirada en el pensamiento verdaderamente igualitarista de François Poulain de la Barre (filósofo cartesiano de finales del siglo XVII) «el feminismo - avant la lettre- era un verdadero test de filosofía, de honestidad epistemológica y de autoexigencia ética y cultural» que muy pocos filósofos van a poder pasar. Las mujeres que soñaron en su momento con la igualdad que les prometía la ilustración (Mary Wollstonecraft u Olympe de Gouges) chocaron frontalmente con la negativa de sus compañeros ilustrados a llevar la universalización de la razón y los derechos que de ella se derivan al sexo femenino. Las ansias por la igualdad de derechos para las ciudadanas recibieron pronto carpetazo con la naturalización de los sexos que según «insignes ilustrados» impedía pensar en un sujeto mujer con plena libertad y autonomía como el sujeto hombre; sujeto, este último, del cual y para el cual la filosofía ilustrada teorizaba la igualdad. Veamos cómo expresa esto Amelia Valcárcel en una clarificadora cita:

"La idea de igualdad estaba disponible con su enorme potencia. El feminismo se la apropió. Entonces sabemos que a la vindicación de igualdad se respondió con la naturalización del sexo. Que las mujeres fueran sexo dominado era designio de la naturaleza, orden inalterable, condición prepolítica, para cumplir la cual también era útil que se les impidiera el acceso a la educación y se les prohibiera el ejercicio de toda profesión. Así se comportaron los primeros democratismos, así lo entendieron

3. Véase en sus obras de 1991, 1993, 1997 y 2000, referenciadas al final de este artículo en la sección de bibliografía.

4. Véase en sus obras de 1990, 1992 y 2000, referenciadas al final de este artículo en la sección de bibliografía.

5. En Tiempo de Feminismo. Sobre feminismo, proyecto ilustrado y postmodernidad, Madrid, Cátedra, 1997, p. 162. 
los fundadores de la filosofía política moderna. Pero la idea de igualdad es pertinaz, incluso cabezota. Ahí seguía disponible y quienes la usaban cada vez tenían mayores dificultades para ponerle fronteras" ${ }^{6}$.

Deconstruir esta naturalización y esencialismo ha supuesto prácticamente el trabajo de casi dos siglos de feminismo (pertinaz y cabezota). Las fronteras se han movido finalmente, pero para ello ha sido necesaria una larga lucha de muchas mujeres y algunos hombres en todos los frentes.

La revisión feminista del corpus filosófico que se viene realizando de manera intensa principalmente desde los años 70 se ha centrado mayoritariamente en tres tipos de tareas:

1. Revisión de la producción filosófica de los hombres a lo largo de la historia de la filosofía, con los hallazgos consecuentes de posturas contradictorias y de lo que graciosamente Celia Amorós denomina "perlas misóginas».

2. Estudio de la historia con el interés de buscar los textos y las experiencias de las mujeres, normalmente invisibilizadas y ocultadas por la historia oficial. Es lo que por ejemplo Elaine Showalter ${ }^{7}$ ha denominado "ginocriticismo" (gynocriticism).

3. Construcción y reconstrucción de discursos filosóficos feministas que forman principalmente lo que se llama actualmente "teoría feminista», compuesta por una amplísima variedad de discursos y filosofías.

Podemos decir que la teoría feminista supone la revisión más contundente de lo que se llama el "canon filosófico», el cual pese a las enormes diferencias en los discursos mantiene un cierto punto de homogeneidad cifrado en el concepto de sujeto que está a la base de las filosofías oficiales, es decir el sujeto varón.

Reescribir lo que llamamos pues el canon filosófico va a convertirse en objetivo prioritario de la investigación filosófica feminista. Las filósofas feministas criticarán principalmente dos exclusiones del canon:

-la exclusión histórica de las mujeres de la tradición filosófica, y

-la caracterización negativa de las mujeres (o lo femenino) en esa tradición.

Así, como ha señalado Charlotte $\mathrm{Witt}^{8}$, el proyecto de incluir a las mujeres en el "nosotros» filosófico es tanto factual como conceptual. La filosofía ortodoxa (y su canon) no sólo es incompleta, porque omite a las mujeres filósofas, sino sesgada, porque devalúa y desprecia a las mujeres y sus pensamientos, que voluntariamente omite. Pero además, y en mi opinión, el feminismo supone una tarea de reconstrucción de lo que la misma filosofía es ${ }^{9}$. Las mujeres

6. VALCÁRCEL, Amelia: "Las filosofías políticas en presencia del feminismo», en Celia Amorós (ed.): Feminismo y Filosofía, Madrid, Síntesis, 2000, pp. 115-133. La cita es de esta obra en página 116.

7. Ver en SHOWALTER, Elaine (ed.): Speaking of Gender, London, Rotledge, 1989.

8. Véase en "How Feminism is Re-writing the Philosophical Canon". Conferencia "The Alfred P.Stiernotte Memorial Lecture in Philosophy" en Quinnipiac College, USA, 2 de Octubre de 1996.

9. En realidad soy de la opinión de que toda filosofía particular es ya una pretensión de repensar lo que la misma filosofía como tarea es. 
filósofas han argüido que la tradición filosófica ha fallado estrepitósamente al estar basada en un concepto de razón que en su misma base está generizado. El canon filosófico occidental no ha cumplido por ello con uno de los aspectos más principales del pensar filosófico: su voluntad de totalidad. El sujeto filosófico en su mismo origen nace ya de sí definido, centrado e identificado en un tipo particular individual, que ni siquiera en la época de más fervor igualitarista, la ilustrada, se muestra abierto a fracturas. Esto demuestra claramente la capacidad acrítica que también la filosofía, como otras disciplinas, es capaz de cultivar, pese a su horizonte y su ideal. Aunque bien es cierto que la solución a ello no será sino "más filosofía». Los prejuicios tampoco escapan a la disciplina filosófica. Algunos de esos prejuicios se muestran prácticamente repetidos en las obras de los que se consideran los más insignes pensadores de la filosofía occidental. Deshacer algunos de esos prejuicios es lo que ha venido haciendo la filosofía feminista (o el feminismo filosófico) ${ }^{10}$ desde la Ilustración (a la que respondió con la misma receta, «más Ilustración»). Sólo en el siglo XX la filosofía depondrá "ese» sujeto "neutral», aunque para entonces esa misma filosofía (ahora postmoderna) estará mayoritariamente dispuesta a enterrar cualquier pretensión de construcción de un sujeto realmente universal. Como veremos más adelante, frente a esta crisis de la razón y del sujeto la crítica feminista planteará bien la conclusión de que tenemos que renunciar a la razón como proyecto filosófico (feminismos de la diferencia y feminismos postmodernos) o bien que la razón muestra más que nunca la necesidad de ser reconceptualizada por los feminismos (feminismos de la igualdad).

Como hemos dicho, esta empresa de revisión feminista se aglutina principalmente en los últimos 30 años, aunque podemos encontrar en la historia de la filosofía, precisamente gracias a la labor de "rescate», voces discordantes de mujeres esparcidas desde la misma época griega. En los siglos XVIII y XIX esas voces dejan de ser puras excepciones para entrar a engrosar lo que hoy llamamos la historia del feminismo filosófico (o de la filosofía feminista).

10. Celia Amorós mantiene que el nombre de «filosofía feminista» no es adecuado, pues "parece connotar un quehacer constructivo y sistemático que, de hecho, estamos aún en condiciones precarias de abordar: sin contar con los problemas que en la actualidad plantea hacer filosofía sistemática en general, feminista o no" (Feminismo y Filosofía, Madrid, Síntesis, 2000, p. 10). Frente a esa acepción ella propone la de "feminismo filosófico", pues ciertamente el feminismo sí es susceptible de ser tematizado filosóficamente. Creo que de todas las razones que Amorós da, la principal para esta opción frente a la primera está en la misma idea de que«a la filosofía qua tale quizás no sea pertinente adjetivarla» (Op.cit., p. 9). Yo por mi parte comparto (aunque con ciertas dudas que ahora no es momento de desarrollar) estas argumentaciones, aunque entiendo que ello también debiera llevarnos a renombrar gran parte de lo que hasta ahora se ha llamado simplemente «ilosofía»a secas (o pura, como en otras épocas se ha denominado) y pasar a llamarla "filosofía androcéntrica»; incluso a veces podría nombrarse "filosofía misógina» o, como algunas filósofas han propuesto, "filosofía pre-feminista»; es decir, filosofía claramente adjetivada. Repito mis dudas frente a esta polémica y espero poder en un futuro profundizar sobre este tema, pues realmente no es un simple problema de nombre sino de entender qué es la filosofía y qué es el feminismo. 
En el siglo XVIII y con la Ilustración se da la primera crítica contundente al subterfugio del universalismo occidental que solapa lo humano con los varones blancos. Como hemos dicho más arriba, es precisamente en el momento histórico y filosófico en que el concepto de igualdad se convierte en eje central de pensar lo humano cuando se reivindicará por parte de las mujeres su inclusión en el grupo humano y de ciudadanía con plenos derechos.

En el siglo XIX, el siglo de los movimientos sociales de emancipación, el feminismo aparece por vez primera como un movimiento social de liberación. Es un movimiento con una identidad teórica y organizativa suficientemente autónoma que permitirá la lucha sufragista por la consecución del voto de las mujeres. Este movimiento de finales del XIX es el que se cataloga normalmente como "primera ola del feminismo" aunque, atendiendo a la investigación que las componentes del grupo del Seminario Permanente "Feminismo e Ilustración» han llevado a cabo sobre las voces vindicantes de las mujeres de la Ilustración, sería más justo con la historia -y las feministas debemos empeñarnos en ello- denominar "primera ola del feminismo» a la época Ilustrada, "segunda ola del feminismo» al movimiento sufragista de mujeres del XIX, y finalmente «tercera ola del feminismo» al feminismo de los 70 en adelante. Como argumenta Celia Amorós a este respecto:

«En realidad, y ponemos en ello todo nuestro énfasis, la llamada 'segunda oleada', como denominan al feminismo de los setenta quienes se acuerdan de que existió el sufragismo, es la tercera. La primera oleada, si se la quiere llamar así, tuvo lugar en la Revolución Francesa. Y si nos retrotraemos a ello no es por prurito alguno de erudición histórica, sino porque, como es sabido, en la historia todo criterio de periodización es tributario de una concepción »11.

Siguiendo pues esta periodización en la primera y segunda ola, la tarea feminista desde la filosofía se cifrará en apuntar a la contingencia de la masculinidad de la razón. Como ha señalado la filósofa australiana Genevieve Lloyd ${ }^{12}$ la filosofía desde Platón a Simone de Beauvoir revela claramente que la razón en el canon filosófico se ha ido construyendo y asociando a la masculinidad. Los feminismos de las primeras épocas pretenderán que la filosofía, como corpus teórico, ha de admitir que la capacidad de razonar es neutral respecto del sexo, aunque ello no implique que no haya diferencias racionales que puedan ser explicadas por diferencias corporales.

El concepto básico del feminismo, desde sus orígenes, podemos decir que es el de igualdad. En concreto el feminismo tiene como principio fundante la idea de que hombres y mujeres cuentan con las mismas capacidades esenciales y que, por tanto, ello debe llevar a poder disfrutar de las mismas oportunidades. Es cierto que también el feminismo, desde lo que se considera sus inicios a finales del siglo XIX con la lucha organizada de manera colectiva por las mujeres

11. Véase en «Presentación que intenta ser un esbozo del status questionis», en Celia Amorós (ed.): Feminismo y Filosofía, Madrid, Síntesis, 2000, pp. 9-112. La cita en la página 22.

12. Véase The Man of Reason: "Male» and "Female» in Western Philosophy, London, Methuen, 1984. 
para pedir el voto, acepta las diferencias entre hombres y mujeres en algunos ámbitos, pero lucha porque esas diferencias sean reconocidas en igual valor a aquellas diferencias de los hombres. Por ello también la aceptación de diferencias se hace y se exige dentro de un marco conceptual que tiene la igualdad como concepto rector.

La tensión entre los diferentes feminismos se genera cuando hay que explicar en qué consisten esas diferencias y qué alcance tiene el marco de igualdad que vaya a servir de trasfondo para la interpretación feminista del mundo. Este será de hecho uno de los temas prioritarios de la agenda de la tercera ola (la que empieza en los 70).

\section{LA TEORÍA FEMINISTA. EL SISTEMA SEXO/GÉNERO}

A partir de los años 70, y con el impulso de los movimientos liberatorios de mujeres que se conocen como "segunda ola del feminismo" y que aquí estamos denominando "tercera ola», la cuestión de la igualdad y la diferencia pasa a colocarse en un lugar central de la dialéctica feminista. Si, por un lado, la igualdad es un objetivo claro que ha dado sus frutos en los campos legal, político y tímidamente en el laboral y económico, por otro la aspiración igualitaria es problemática porque puede reproducir (y de hecho muchas veces reproduce) la norma de los hombres, tanto en contenido como en formas. En cierta manera podemos decir que a medida que la lucha por la igualdad de oportunidades entre sexos ha alcanzado algunos éxitos, el discurso de la diferencia ha ido ganando más terreno en la teoría feminista.

Veamos ahora cuál es el debate filosófico de la teoría feminista contemporánea.

\subsection{La teoría feminista: el concepto de género}

La teoría feminista se define normalmente como la producción teórica que se origina en el feminismo. Qué es el feminismo puede llegar a resultar una cuestión ardua e intrincada. Sin embargo, en este escrito, tomaré la definición de feminismo más amplia y generalizada, y que, por tanto, puede servir de denominador común de todos los feminismos, independientemente de sus diferencias en las luchas y en los planteamientos teóricos. La definición común de feminismo se apoya en dos puntos:

-apreciación y conciencia del hecho de que las mujeres están menos y peor valoradas que los hombres en sociedades que categorizan a hombres y mujeres en diferentes esferas culturales, políticas y económicas.

-propuesta de un deseo activo por cambiar esta realidad.

La posibilidad de una lucha feminista que se extienda a la consecución de sociedades donde las mujeres no sufran subordinación se ha visto propulsada con el concepto de "género». Al igual que cualquier lucha social de liberación de grupos humanos, por ejemplo la lucha contra el racismo, el feminismo ha fundamentado los pasos hacia la liberación de las mujeres poniendo en evidencia cada vez con más fuerza cómo la base de las desigualdades, la subordinación y la dominación de las mujeres no está fijada o determinada de manera pre-social 
(por ejemplo biológicamente), sino construida a través de los órdenes social, económico y político de las sociedades.

Una de las obras seminales del feminismo, El segundo sexo (publicada originalmente en 1949), estableció precisamente el concepto de género a la hora de entender la construcción de la mujer como sujeto subordinado y oprimido. El concepto de género marcó sin ninguna duda la agenda de la tercera ola del feminismo. Con este concepto se hizo claro que la categoría de «mujer» es una construcción social que se hace sobre el cuerpo biológico femenino. De una manera rotunda esto lo expresará la célebre frase de Simone de Beauvoir en su libro El segundo sexo: "No se nace mujer, se llega a serlo.» ${ }^{13}$ Con esto se dejaba atrás una tradición patriarcal que interpretaba que la distinción entre hombre y mujer era una distinción que obedecía a una diferenciación sexual y biológica indiscutible. La biología se había entendido, en célebre frase de Freud, como destino.

Simone de Beauvoir analiza en El segundo sexo el concepto de "alteridad» como una categoría esencial del ser humano. La identidad de la propia subjetividad necesita de la categoría del "otro». El estudio de Beauvoir incide precisamente en cómo ese "otro» se ha estigmatizado y ha pasado a convertirse en un sujeto objetivizado, en un absoluto. Consecuentemente la mujer se convierte en alteridad absoluta cuando se construyen las leyes contra ella; leyes que buscan proteger los intereses económicos, políticos, morales y ontológicos del sujeto que las crea: el varón.

Durante siglos las teoría naturalistas para apoyar y mantener la subordinación de las mujeres, pretendían convencer de que ésta no ha "devenido", no es un acontecimiento, no es un hecho histórico, sino que se presenta como «natural».

El concepto de género deja claro, en cualquier campo de conocimiento, que la subordinación y opresión de las mujeres se deben a una construcción social y no natural ni biológica. La importancia del concepto de género se hace patente si pensamos que no hay opresión comparable a de las mujeres, pues al biologizarse se convirtió en un proceso natural, ahistórico y más allá de la propia voluntad humana. Se hace urgente pues una tarea de desnaturalización de las bases del patriarcado si no se quiere aceptar que los logros feministas han llegado a su fin con la consecución del voto y unos tímidos derechos laborales. Precisamente es la consecución de ciertos logros en la igualdad lo que lleva a la agenda feminista a plantearse nuevos retos. Como ha señalado María Jesús Izquierdo en El malestar de la desigualdad ${ }^{14}$,

13. La célebre frase fue originalmente de François Poulain de la Barre, cartesiano de finales del siglo XVII del cual Beauvoir recoge la cita que sirve para abrir su libro. Con ello se muestra, como han advertido repetidas veces las mujeres que formaron el Seminario Permanente de "Feminismo e Ilustración» liderado por Celia Amorós, que el feminismo hunde sus raíces en la Ilustración.

14. El malestar de la desigualdad, Madrid, Cátedra, 1998, p. 18. 
«Primero las mujeres luchan contra la discriminación sexual, sólo después recibe atención en la comunidad científica el hecho de que las diferencias físicas no generan la desigualdad sexual, sino que la soportan. La separación analítica entre sexo y género se realiza después de que las mujeres han demostrado prácticamente que ser hembra de la especie no es una dificultad para ser ingeniera, o conductora de autobús, o para ser autónoma económicamente» ${ }^{15}$.

El salto entre el sexo y el género es precisamente uno de los temas principales de la teoría feminista. El potencial constructivista del concepto de género será de norme envergadura para la filosofía feminista, pues supondrá que podemos introducir cambios en la estructura epistemológica, que podemos finalmente repensar cómo construir la feminidad y también la masculinidad. Cómo en definitiva se construye el otro y, en consecuencia, las bases de su exclusión. Podemos así resumir de momento que la teoría feminista es la teorización de la construcción del "otro» (en este caso "otra») como sujeto dominado.

El feminismo se viene señalando en los últimos años como el movimiento social más importante del siglo XX. Su importancia radica en la cantidad de cambios que ha generado en todas las esferas de las sociedades, principalmente y de manera patente en las sociedades occidentales. La visión usual del feminismo como un movimiento "radical» puede ser interpretado precisamente como una señal del potencial crítico y de cambio de estructuras que se le adjudica. Es más, la diversificación de planteamientos teóricos que la teoría feminista ha recibido en las dos últimas décadas, es una muestra clara de que estamos ante reflexiones críticas y filosóficas, y no tanto ideológicas.

La importancia del feminismo como movimiento crítico de transformación social nos revela de inmediato su naturaleza tanto teorética como práctica. Se trata así de un "proyecto teórico comprometido con producir análisis críticoconstructivos de las estructuras sistémicas de poder, presuposiciones teóricas, prácticas sociales, e instituciones que oprimen y marginalizan a las mujeres y afectan una transformación social» ${ }^{16}$. Es decir, que el feminismo es tanto una teoría (crítica) como una práctica. Y por ello la teoría feminista supone un balance con la práctica, y promueve realmente un más allá de la dicotomía teoría y práctica, ya que esta misma separación obedece realmente a una razón patriarcal. El feminismo es, como Celia Amorós ha expuesto, una "pragmática de la razón».

15. María Jesús Izquierdo distingue en su libro entre «discriminación por sexo» y «desigualdad de género». La primera se manifiesta en general en el hecho de que «las hembras, ocupen posiciones sociales femeninas o masculinas, están peor pagadas, consideradas, o tienen menor poder que los machos en las mismas posiciones de género que ellas»(pp. 51-52). La desigualdad de género en cambio «se produce tanto si las personas que desarrollan estas actividades son hembras como si no lo son. Manifestaciones de la desigualdad estructural de género son el hecho de que el nivel retributivo, formativo, de prestigio y/o de poder para las actividades femeninas es inferior al que se le otorga a las actividades masculinas, al margen de que quienes las desarrollan sean hembras o machos»(p. 51). Pese a la distinción conceptual, es claro ver que normalmente una y otra, discriminación sexual y desigualdad de género, van asociadas.

16. En CODE, Lorraine (ed.): Encyclopedia of Feminist Theories, London, Routledge, 2000, p. XIX. 
La consciencia de una necesidad teórica que impulse la necesaria transformación social no es, sin embargo, parte importante de las primeras etapas del feminismo. Es en la tercera ola del feminismo (comúnmente denominada segunda ola) que se generó de manera cada vez más clara y concisa una consciencia de la necesidad de desarrollar como tarea principal del feminismo análisis críticos de las estructuras de las sociedades patriarcales y del patriarcado como sistema de poder. El objetivo de esta revuelta crítica es cambiar y transformar las sociedades patriarcales, pero para ello es necesario estudiar y teorizar críticamente sobre las mismas.

Si es a través de la producción de conocimiento como se ha ido fundamentando el patriarcado habrá de analizarse tal desarrollo para poder alcanzar un estadio tal en que podamos subvertir la forma en que el conocimiento sirve a la dominación de las mujeres.

La consecución del voto y de ciertas mejoras sociales para las mujeres (objetivo principal de las feministas sufragistas) se mostró claramente insuficiente a mediados del siglo XX. La lucha de las sufragistas había sido ardua, pero aun así se mostraba insuficiente.

La teoría feminista empieza precisamente cuando se hace necesario reflexionar sobre las razones de esa insuficiencia. Cuando se hace necesario interrogarse sobre la desigual distribución de poder y privilegios; subyugación de las mujeres en prácticamente todas las sociedades conocidas.

La teoría feminista, por tanto, es una tarea no sólo oportuna, sino totalmente necesaria para poder cambiar la situación de las vidas de las mujeres. Siguiendo a Lorraine Code:

"La teoría ocupa un lugar central en los movimientos feministas, pues las experiencias no hablan por sí mismas: por esta razón la concienciación ha sido un componente vital de la práctica feminista desde el principio de la segunda ola [que aquí llamamos tercera]. Las mujeres han tenido que aprender de cada una cómo se nombran sus experiencias; cómo reconocer y evaluar sus similitudes y diferencias. La teoría -y la concienciación- permanecen fundamentales para las primeras asumciones sobre igualdad femenina (sororidad), de definir y redefinir cuestiones centrales, y de activar nuevas estrategias transformativas en respuesta a la evolución, y a menudo regresión de las circunstancias socio-políticas ${ }^{17}$.

Como el largo camino de lucha feminista evidencia, el patriarcado está compuesto de múltiples prácticas que, tomadas una por una, pueden parecer insignificantes o no opresivas pero que juntas (reforzándose unas a otras) conforman una estructura sistemática omnipresente y casi inabordable.

Los análisis teóricos feministas han ayudado a deconstruir algunos de los mitos más esencialistas tanto del patriarcado como de algunas posiciones del mismo feminismo.

Después de más de 30 años de teoría feminista, la misma conciencia que empujaba por entender los intersticios de las relaciones de poder que se confor-

17. En CODE, Lorraine (ed.), op. cit., p. XIX. 
man en las estructuras del patriarcado, nos ha llevado a la conciencia de la diferencia. Si bien las mujeres son subyugadas, no todas lo son de la misma manera ni bajo las mismas circunstancias. Razones de clase, raza, sexualidad, etnicidad, religión, ideología, profesión, etc., complican el panorama de las relaciones de poder y dominación. Y por ello cada vez se hace más difícil hablar de experiencia genérica de las mujeres. Estos son precisamente los aspectos que han abierto una nueva etapa en el feminismo, la que de momento llamamos «cuarta ola» (y que en terminología internacional estándar es «tercera ola»).

Veamos ahora a grandes rasgos los debates actuales en teoría feminista.

\section{LOS DIFERENTES ENFOQUES TEÓRICOS DEL FEMINISMO: LOS FEMINISMOS}

\subsection{El feminismo de la diferencia frente al de la igualdad}

La igualdad se ha convertido en un concepto altamente controvertido dentro del feminismo. El concepto de igualdad que surgió en las luchas feministas de la primera y segunda olas ha sido acusado posteriormente por algunos feminismos contemporáneos como un obstáculo para las diferencias. Ciertamente los problemas a resolver son los mismos, es decir, la cuestión del poder, la separación de las esferas privada y pública, la cuestión del trabajo, y la resignificación de conceptos que aún perviven dominados por estructuras patriarcales. Detrás de todos esos problemas permanece también un núcleo que podríamos llamar "duro", y que es el hueso a roer por toda feminista, sea de la ideología que sea y tome la perspectiva que tome: el de transformar el ámbito cultural y simbólico en otro que no implique dominación sobre las mujeres y permita a éstas libertad y subjetividad plena. Cómo hacerlo es básicamente el punto donde empiezan los diferentes enfoques.

El llamado «feminismo de la diferencia» engloba etiquetas como «feminismo de la diferencia sexual", "feminismo cultural», "affidamento" si nos referimos al feminismo italiano de la diferencia que a partir de los 60 luchaba por un reconocimiento de la autoridad femenina, feminismo francés de la "écriture femenine», o el también francés de los 60 de «Psychanalyse et Politique»" Detrás de todos estos posicionamientos teóricos, y algunos más que no recogemos, hallamos la demanda feminista de variar el modelo de vindicación feminista; el objetivo ya no es conseguir la igualdad, puesto que para las mujeres "ser igual» acabará convirtiéndose en "ser iguales a los hombres», y ello -se dice- porque por una parte anula la subjetividad "femenina» (al moldearla sobre el modelo masculino) y por otra impide la verdadera liberación de las mujeres.

Dado el panorama de multiplicidad de voces en el feminismo de la diferencia es difícil actualmente agrupar sus propuestas. Sin embargo, y sin pretender

18. Para una categorización clara y esquemática de los diferentes feminismos ver Ana De Miguel: «Feminismos»en Diez palabras clave sobre Mujer, Celia Amorós (directora), Pamplona, Editorial Verbo Divino, 1995. 
ser una clasificación completa, seguimos el análisis de Kate Nash ${ }^{19}$, según el cual podemos hablar de tres escuelas feministas del pensamiento de la diferencia:

1. El pensamiento maternalista, para el cual la diferencia entre hombres y mujeres es básicamente de orden moral. Carol Gilligan y Nancy Chodorow son dos exponentes de esta postura. Para estas autoras el núcleo de la identidad femenina y de su desarrollo psíquico está en la relación temprana con la madre. El diferente cuidado y relación que la madre tiene con su progenie si ésta es de un sexo u otro provoca las diferencias de pensamiento, psíquicas y morales entre hombres y mujeres. Como consecuencia de ello estas autoras afirman que las mujeres desarrollan un pensamiento más relacional, mientras que el de los hombres es más unidireccional y lógico.

2. Las teóricas postestructuralistas de la differance, para las cuales lo femenino es de sí tan particular que no se puede representar. Agrupadas prioritariamente por lo que se conoce como "teóricas feministas francesas» afirman que incluso la diferencia por la que abogan las maternalistas está dentro del discurso y representación patriarcal. Por ello su propuesta es no representar la identidad femenina, ya que toda representación será falocéntrica y masculina. Lo femenino no puede ser capturado en ninguna representación, pues si lo es entra en un conjunto de categorías y conceptos dominados por la razón dominante, la razón patriarcal. El potencial subversivo de la identidad femenina radica precisamente en mantenerse "descategorizada", en alejarse de las clasificaciones solidificantes del pensamiento patriarcal. Luce Irigaray, Hélène Cixous, y Julia Kristeva, pese a sus diferencias, comparten esta posición.

3. Aquellas que piensan que lo importante son las diferencias múltiples que atraviesan la diferencia sexual. Se oponen a una representación esencialista de las mujeres y argumentan que el proyecto feminista en realidad lo que tendría que hacer es desmantelar el concepto de «mujer». Con ello el feminismo conseguiría su objetivo más importante, deconstruir el de «varón». Pero para llegar a ello autoras como Judith Butler creen que es necesario acabar con la «matriz heterosexual», según la cual el deseo masculino ha de tener como objeto a la mujer femenina, y el deseo femenino ha de tener como objeto al hombre masculino. La propuesta lleva a una proliferación transgresora de identidades sexuales y de sexualidades múltiples y cambiantes.

Creo que en estas propuestas ciertamente hay una preocupación por una agenda de liberación de la mujer, y que sin duda la deconstrucción de representaciones de lo femenino es parte importante para ese objetivo. Ahora bien, como cuestionamiento para este tipo de enfoque y con la preocupación de seguir encontrando estrategias políticas que podamos aplicar para acercarnos a esa liberación, ¿quién es el sujeto político de esa lucha? Si no hay un «sujeto mujer», ¿cuáles son las posibilidades de las mujeres de luchar por sus derechos sin perderse en un relativismo que sólo beneficia a aquellos que no necesitan de un

19. En Universal Difference: Feminism and the Libertal Undecidability of 'Women', London, Palgrave Macmillan, 1998. 
discurso universalista porque ya lo tienen todo? ¿Qué es «lo femenino» sin las mujeres?, como ha apuntado acertadamente Françoise Collin. En contrapartida, como responden las feministas de la diferencia, ¿no es el «sujeto mujer» el sujeto que está construido a la vez que dominado? ¿No será mejor por tanto descentrar los conceptos de «mujer»y "hombre», ya que son sólo los posicionamientos identitarios que sirven como base para el reparto de poder y de exclusión?

Como vemos las preguntas no son sólo múltiples, sino realmente fundamentales y de primer orden desde ambos lados de los dos feminismos (conocidos como el de la igualdad y el de la diferencia).

En una interpretación amplia de las diferentes posiciones teóricas feministas y con una mirada penetrante a las actuales luchas de las mujeres, creo que podemos decir que todo discurso feminista mínimamente comprometido está preocupadamente interesado en acabar también con el binarismo entre igualdad y diferencia.

Desde el feminismo de la diferencia el esquema de la igualdad se muestra actualmente inadecuado para tratar la subordinación de las mujeres. Y desde el feminismo de la igualdad no hay vindicación posible sin apelar a la igualdad -como Celia Amorós repetidamente ha contestado ${ }^{20}$, es más, a una igualdad con pretensiones universalistas. Ahora bien, este no es un universalismo al uso, pues siguiendo el concepto de "mentalidad amplia» de la filósofa y feminista Seyla Benhabib ${ }^{21}$ se entiende que no hay valores universales, sino una moralidad universalística, según la cual es necesaria una práctica de renegociación continua que incluso ha de llevar a la habilidad para distanciarse también de las creencias más profundas de una misma ${ }^{22}$. Ello está en sintonía con la concepción de un feminismo de la igualdad que, como Nancy Fraser ${ }^{23}$ reclama, no suponga una narrativa fundacional.

Hemos dicho que para las feministas de la diferencia la igualdad anula la subjetividad femenina pues calza a las mujeres con el modelo de subjetividad masculina; para las feministas de la igualdad precisamente el feminismo debe luchar porque las mujeres puedan constituirse una nueva subjetividad que no sea la de la dominación o exclusión, ni la del varón.¿Cómo encontramos la liberación de los sujetos, que es en definitiva la búsqueda común de ambos feminismos? ¿Dónde hallar ese punto de encuentro?

Para mí es de gran inspiración el trabajo que entre nosotras en España llevan a cabo mujeres que intentan de alguna manera ese punto de encuentro, como la filósofa Fina Birules, quien dice:

20. Ver especialmente en Tiempo de Feminismo. Sobre feminismo, proyecto ilustrado y postmodernidad, Madrid, Cátedra, 1997, p. 71.

21. Ver en: "Political Membership in the Global Era», en Social Research, vol.66, n.3. (Fall 1999), p.729.

22. Para un estudio de este punto ver Sonia Reverter: «Feminismo y democracia. Una crítica antifundamentalista», en Recerca. Revista de Pensament $i$ Anàlisi, Universitat Jaume I, 2001.

23. Ver en Unruly Practices: Power, Discourse and Gender in Contemporary Social Theory, Cambridge, Polity Press, 1989. 
"Quiero decir que del recordatorio de la fragilidad de las conquistas de la igualdad no se sigue, en mi opinión, un desentenderse de ellas, sino que se trata, además, de afrontar lo que se ha dado en llamar el malestar de la emancipación que indica que el progreso en la igualdad de derechos no parece haberse presentado acompañado de la posibilidad de decir las diferencias, y entre ellas, la central es la diferencia de los $\operatorname{sexos}(\ldots)$

En síntesis y para terminar, la igualdad tiene que ver con nuestro estatuto de sujetos de derecho, de ciudadanas, pero no agota el campo de lo político: la igualdad es la condición previa de lo político. De modo que en esta propuesta hay que habérselas con la libertad, con la posibilidad de 'decir' la diferencia sin por ello generar desigualdad.» ${ }^{24}$

A ese «decir la diferencia sin generar desigualdad» es a lo que gran parte del corpus de la teoría feminista se está dedicando en la última década ${ }^{25}$. Uno de los frentes en los que se trabaja es la búsqueda de un concepto de igualdad que a su vez sea sensible a las diferencias. El concepto de «igualdad compleja», sobre el cual trabajan autores como Michael Walzer ${ }^{26}$, Iris Marion Young y otros, puede ser un ejemplo de ello.

\subsection{El post-feminismo}

Podemos afirmar, y creo que por lo que llevamos dicho, con un amplio consenso, que el proyecto feminista como tal es un proyecto que forma parte de la historia de la modernidad.

La cuestión será ahora ver la evolución de ese proyecto en una época en que gran parte de los pilares fundamentantes de la modernidad están firmemente contestados. En un mundo con complejidades sólo entendibles desde los presupuestos postmodernos, con una globalización en ciernes que nos reta a la vez que nos amenaza, y con la reemergencia de formas pre-modernas de fundamentalismo y tradicionalismo, el feminismo ha diversificado enormemente su agenda y sus perspectivas. De hecho es ya común hablar de "feminismos» en plural en vez de "feminismo» en singular para así nombrar la diversidad de planteamientos.

Si bien en la década de los 80 florecen los discursos del feminismo de la diferencia, como hemos visto, la década de los 90 va a cuestionar directamente el concepto de género y el sistema sexo/género organizado sobre él.

En 1975 Gayle Rubin ya incidió en que la cuestión constructivista que el género abre tiene un alcance que llega a la misma sexualidad. Así, según sus

24. En "El sueño de la absoluta autonomía: Reflexiones en torno a la igualdad y a la diferencia», en Amparo Gómez Rodríguez y Justine Talln (eds.), La construcción cultural de lo femenino, Universidad de La Laguna, Centro de Estudios de la Mujer, 1998, p. 29.

25. Para la cuestión que intenta integrar el pensamiento de las diferencias y el postmoderno en el feminismo ver FLAX, Jane: Thinking Fragments. Psicoanalysis, Feminism and Postmodernism in the Contemporary West, Berkeley, University of California Press, 1990.

26. Para un análisis de las implicaciones del concepto de«igualdad compleja»de Michael Walzer para la teoría feminista, ver Chris Armstrong: "Complex Equality: Beyond Equaliy and Difference», en Feminist Theory. An International Interdisciplinary Journal, Vol. 3, nº 1. (1. Abril 2002). 
propuestas, que reinterpretan las tesis antropológicas de Lévi-Strauss sobre la exogamia de las mujeres, no sólo el género es construido, sino también la sexualidad, y precisamente a través del género.

En la década de los 90 retomando de alguna manera esta profundización en el constructivismo la americana Judith Butler con su revolucionario libro Gender Trouble (publicado originalmente en 1990) afirma que también el sexo es construido. La filosofía constructivista de los 90 incorporará cada vez de manera más clara y contundente la identidad como "artefacto» básicamente construido en todas sus dimensiones y coordenadas. Así, siguiendo al pensamiento constructivista francés y al psicoanálisis, algunas teóricas feministas, como la misma Judith Butler, rompen la distancia que el sistema sexo/género mantiene en cierta manera entre el sexo biológico y el género social. Para Butler este planteamiento es absolutamente reductivo y simplista, y lleva a una falsa estabilización que sólo está ayudando a los intereses de la construcción heterosexual. Y ésta es esencialmente la que impone la «ley de los sexos» que sirve para reprimir a los individuos a través de la normativización de la sexualidad y la matriz de inclusión/exclusión con ella asociada, aliadas de primer orden del patriarcado. ${ }^{27}$

Así se abre la revisión al sistema sexo/género al entender que el género, como producto de la cultura, no lleva necesariamente a la liberación; es más, el género puede ser en realidad uno de los mecanismos por los que seguir regulando el comportamiento de hombres y mujeres. En definitiva, las pautas «aceptables» para mujeres y para hombres se siguen produciendo «social y culturalmente» en base a un sexo, que se dice biológico, pero que se moldea realmente en conjunción con el género. Hay por tanto que «desestabilizar» el tándem sexo/género y el binarismo biológico y social alineado con él. Tanto los sexos como los géneros pueden ser no sólo múltiples, sino variantes; sólo un entramado patriarcal que crea un género sobre una supuesta naturaleza biológica sexual dicotómica está convirtiendo en esencial y «natural» la distinción de dos sexos.

A ésta y a otras propuestas feministas entroncadas con la filosofía post-estructuralista y con la postmodernidad le han salido, como a las feministas de la diferencia, preguntas que desde el feminismo de la igualdad se consideran irrenunciables en cualquier agenda feminista de liberación. Una vez más estas cuestiones irán dirigidas a la constitución del sujeto.

Tania Moldeski, en su libro de 1991 oportunamente titulado Feminism without Women: Culture and Criticism in a "Postfeminism" Age, alerta de los peligros de acabar privando a las mujeres de medios significativos para formular una agenda basada en sus experiencias. Si bien es cierto que el antiesencialismo aportó una importante base para la lucha feminista por la igualdad, ello no debe acabar llevándonos a la negación de cualquier posibilidad de generalización de demandas políticas de las mujeres. Es decir, que frente a posturas post-estructuralistas que

27. Para una discusión de las propuestas de Judith Butler ver Sonia Reverter: «Actos de habla y feminismo», en Actas del XIV Congreso de Filosofía del País Valencià, 2002. 
ya han anunciado la muerte del sujeto, la pregunta de Moldeski, que da título a su libro, es: ¿puede haber un feminismo sin mujeres? ¿cómo seguir teorizando el feminismo sin sujeto?

En definitiva, y esta es la crítica más pertinaz a la que deben enfrentarse las feministas postmodernas, la reivindicación de la resistencia y subversión que muchas teóricas postestructuralistas postulan̨quién la hace, si no hay sujeto?, como pregunta Alicia Puleo ${ }^{28}$.

\section{UNA PREGUNTA PARA SEGUIR PENSANDO}

¿Hacen las mujeres una filosofía diferente?:Hay una voz feminista en filosofía, como nos pregunta el título de Janet Kourany, Philosophy in a Feminist Voice?

Como Susan Bordo ${ }^{29}$ ha señalado, las feministas que estamos en filosofía no sólo tenemos que apuntar nuestros dardos a lo que la filosofía ortodoxa ha excluido, sino también a lo que ha incluido. En este sentido hay, en muchas filósofas feministas, la idea de que lo pensado y tematizado por los varones en la filosofía hubiera sido muy diferente si lo hubiesen hecho las mujeres. Genevieve Lloyd ${ }^{30}$, por ejemplo, cree, en esta línea de pensamiento, que lo que necesitamos ahora no es la demarcación de una distintiva metodología feminista, sino la continuación de nuestra contribución con «formas imaginativas» tanto al plantear nuevas filosofías como al revisar las históricamente dadas. Lloyd está convencida, por ejemplo, de que la reconstrucción de una filosofía política kantiana por parte de Hannah Arendt, pese a no presentarse como "feminista", aporta nuevas estrategias de lectura e interpretación que sin duda deben mucho a la experiencia de Arendt como mujer. En esta línea econtramos vertientes feministas que se decantan por una nueva hermenéutica ${ }^{31}$ que introduzca los conceptos de género, feminidad, diferencia...

Si la filosofía se construye, como normalmente se dice, como un esfuerzo tenaz de pensar las cosas de otra manera, las mujeres pueden ofrecer mucho para ese cambio: desde nuevas miradas a las filosofías del pasado, pasando por la recuperación de voces silenciadas, y llegando a afrontar problemas filosóficos para pensar el presente y el futuro con nueva y renovada imaginación filosófica ${ }^{32}$.

28. Ver especialmente "Sujeto, sexo y género en la polémica Modernidad-Postmodernidad», en Multiculturalismo y diferencia. Sujetos, nación y género. Anales de la Cátedra de Francisco Suárez, n.31. (1994). Y Filosofía, Género y Pensamiento Crítico, Universidad de Valladolid, Secretariado de Publicaciones, 2000.

29. En «The Feminist as Other», en Janet A. Kourany (ed.): Philosophy in a Feminist Voice. Critiques and Reconstructions, Princeton University Press, 1998.

30. En «Feminism in History of Philosophy», en Miranda Fricker y Jennifer Hornsby(eds.), Feminism in Philosophy, Cambridge University Press, 2000, pp. 245-263.

31. El grupo de «Filosofía y Género»de la Universidad de Barcelona, coordinado por Fina Birulés, trabaja con esta hermenéutica«feminista»para una recuperación de la memoria.

32. Es lo que de alguna manera Michèle Le Doeuff en sus exploraciones sobre el «imaginario filosófico»(philosophical imaginary) ha denominado"cambio de pensamiento»(shift thinking). 
Creo sinceramente que las investigaciones filosóficas ortodoxas de las academias no están atendiendo a estas teorizaciones de los últimos feminismos. Y me temo que ello explica en parte la doble agenda investigadora que aún en muchos departamentos de filosofía debemos llevar muchas personas, normalmente mujeres, que nos dedicamos a la investigación feminista. En una crítica reflexión sobre esta cuestión la filósofa Susan Bordo afirma que los proyectos críticos de filosofía con voz feminista han sido percibidos normalmente como estrechamente relacionados con «temas de mujeres», y por tanto opuestos a temas "universales» o «humanos». Detrás de ello, en las instituciones dedicadas a la investigación filosófica sigue habiendo, generalmente por parte de muchos hombres, la idea de que la reflexión crítica es "neutra» respecto del género. La teoría por tanto, no puede implicarse en una variante particular del sujeto universal, las mujeres; eso, en todo caso, habrá de ser objeto de la filosofía práctica. Como resultado, y como denuncia Bordo, tenemos que la filosofía con voz feminista se deja para «los otros» (es decir, se ignora), y sus logros o bien se minimizan o acaban siendo asumidos por las corrientes más ortodoxas. El mérito de las teóricas feministas parece haber sido simplemente hacer visible la construcción del género, y vigilar para que en adelante todo estudio serio incluya de pasada unas líneas sobre el género. Ese parece ser el mísero tributo de los que están dispuestos a reconocer, que por supuesto no son todos, alguna aportación del feminismo a la reflexión filosófica. Ello no deja, sin embargo, de responder a una representación victimista de las mujeres, sean estas enfermeras, empresarias, o filósofas; es decir, una representación que mantiene a las mujeres en la otredad, que en nuestro caso, como Freud ya anunció, es equivalente a ser puro sexo. Sin embargo, quien conozca mínimamente la teoría feminista sabe que la denuncia y la vindicación, que no el victimismo, son nuestras maneras de hacer también filosofía.

No se puede decir que la filosofía feminista se ha centrado en cuestiones periféricas o marginales. Sus reflexiones se vierten sobre el sujeto, su construcción y muerte, la razón, la autonomía, la identidad, el poder...es decir, temas centrales a la reflexión filosófica. Las filósofas feministas están trabajando en conceptos ampliamente discutidos, conceptos de gran potencial y centralidad para la misma filosofía.iPor qué entonces no forma parte de los curricula en las instituciones de educación universitaria en filosofía

Christine Battersby ${ }^{33}$ describe la filosofía como «la disciplina recalcitrante» en resistirse a los debates contemporáneos de la teoría feminista. Pamela Sue Anderson, quien habla desde su experiencia en la academia británica, (la cual cuenta con muchísima más presencia de la perspectiva feminista entre sus departamentos y aulas que la academia española) cree que la razón no es otra que la ignorancia:

33. En The Phenomenal Woman: feminist Metaphysics and the Patterns of Identity, London, Routledge, 1998.

34. En "Feminism and Philosophy», en Sarah Gamble(ed.): Feminism and Postfeminism, Cambridge, Icon Books, 1999, p. 152. 


\section{La perspectiva de género en la filosofía}

«La ignorancia básica del impacto de la filosofía feminista sobre la filosofía general tiende a crear una resistencia al feminismo como algo -erróneamente- temido» ${ }^{34}$

El trabajo por hacer desde la filosofía es, a mi parecer doble: de disidencia, de sentarse en otro campo, de dejar de ser "alteridad absoluta»; y de disensión, de sentir diferente, y tener pleno derecho a esa diferencia.

\section{BIBLIOGRAFÍA}

Amorós, Celia: «El feminismo, senda no transitada de la Ilustración», Isegoría, 1 (1990), Madrid, Instituto de Filosofía, CSIC.

Amorós, Celia: "Notas para una teoría nominalista del patriarcado», Asparkía, 1 (1992), Castellón, Seminario de Investigación Feminista, Universitat Jaume I.

Amorós, Celia: Tiempo de Feminismo. Sobre feminismo, proyecto ilustrado y postmodernidad, Madrid, Cátedra, 1997.

Amorós, Celia: «Presentación que intenta ser un esbozo del status questionis», en Celia Amorós (ed.): Feminismo y Filosofía, Madrid, Síntesis, 2000, pp. 9-112.

Anderson, Pamela Sue: «Feminism and Philosophy», en Sarah Gamble (ed.), Feminism and Postfeminism, Cambridge, Icon Books, 1999.

Battersby, Christine: The Phenomenal Woman: feminist Metaphysics and the Patterns of Identity, London, Routledge, 1998.

Beauvorr, Simone de: El Segundo sexo, Madrid, Cátedra, 1999. Traducido por Alicia Martorell del original francés de Editions Gallimard, Paris, 1949.

BenHAbIB, Seyla: «Political Membership in the Global Era», en Social Research, vol.66, n.3 (Fall, 1999).

Birulés, Fina (ed.): Filosofía y Género. Identidades Femeninas, Pamplona, Palmiela, 1992.

Birulés, Fina: «El sueño de la absoluta autonomía: Reflexiones en torno a la igualdad y a la diferencia», en Amparo Gómez Rodríguez y Justine Talln (eds.), La construcción cultural de lo femenino, Universidad de La Laguna, Centro de Estudios de la Mujer, 1998.

Bordo, Susan: «The Feminist as Other», en Janet A. Kourany(ed.), Philosophy in a Feminist Voice. Critiques and Reconstructions, Princeton University Press, 1998.

Butler, Judith: Gender Trouble, London, Routledge, 1990. Traducido al español por Mónica Mansour y Laura Manríquez, El género en disputa, Barcelona, Paidós, 2001.

Code, Lorraine (ed.): Encyclopedia of Feminist Theories, London, Routledge, 2000.

FlaX, Jane: Thinking Fragments. Psicoanalysis, Feminism and Postmodernism in the Contemporary West, Berkeley, University of California Press, 1990.

Fraser, Nancy: Unruly Practices: Power, Discourse and Gender in Contemporary Social Theory, Cambridge, Polity Press, 1989.

Izouierdo, María Jesús: El malestar en la desigualdad, Madrid, Cátedra, 1998.

Le Doeuff, Michèle: Hipparchia's Choice: An Essay Concerning Women, Philosophy, etc, Oxford, Blackwell, 1991.

Lloyd, Genevieve: The Man of Reason: "Male» and "Female» in Western Philosophy, London, Methuen, 1984.

Lloyd, Genevieve: "Feminism in History of Philosophy», en Miranda Fricker y Fennifer Hornsby (eds.), Feminism in Philosophy, Cambridge University Press, 2000: 245-263.

Kourany, Janet A.(ed.): Philosophy in a Feminist Voice. Critiques and Reconstructions, Princeton University Press, 1998.

Miguel, Ana de: «Feminismos» en Diez palabras clave sobre Mujer, Celia Amorós (directora), Pamplona, Editorial Verbo Divino, 1995. 
MoldeskI, Tania: Feminism without Women: Culture and Criticism in a "Postfeminism» Age, London, Routledge, 1991.

NASH, Kate: Universal Difference: Feminism and the Libertal Undecidability of 'Women', London, Palgrave Macmillan, 1998.

Puleo, Alicia: «Sujeto, sexo y género en la polémica Modernidad-Postmodernidad», en Multiculturalismo y diferencia. Sujetos, nación y género. Anales de la Cátedra de Francisco Suárez, n.31, 1994.

Puleo, Alicia: Filosofía, Género y Pensamiento Crítico, Universidad de Valladolid, Secretariado de Publicaciones, 2000.

ReVERTeR, Sonia: «Feminismo y democracia. una crítica antifundamentalista», en Recerca. Revista de Pensament $i$ Anàlisi, Universitat Jaume I, 2001.

Reverter, Sonia: "Actos de habla y feminismo», en Actas del XIV Congreso de la Societat de Filosofía del País Valencià, 2002.

RuBIN, Gayle: "The Traffic in women: notes on the 'political economy' of sex», en Rayna Reiter (ed.), Toward an Anthropology of Women, Mew York, Monthly Review, 1975.

VAlCÁrCel, Amelia: Sexo y Filosofía, Madrid, Anthropos, 1991.

VALCÁRCel, Amelia: Del miedo a la Igualdad, Madrid, Crítica, 1993.

VAlCÁrCel, Amelia: La politica de las mujeres, Madrid, Cátedra, 1997.

ValCÁrcel, Amelia: «Las filosofías políticas en presencia del feminismo», en Celia Amorós (ed.): Feminismo y Filosofía, Madrid, Síntesis, 2000, pp. 115-133.

WITT, Charlotte: «How Feminism is Re-writing the Philosophical Canon». Conferencia "The Alfred P. Stiernotte Memorial Lecture in Philosophy» en Quinnipiac College, USA, 2 de Octubre de 1996.

Young, Iris Marion: Justice and the Politics of Difference, Princeton University Press, 1990.

Young, Iris Marion: "The Ideal of Community and the Politics of Difference», en Linda Nicholson (ed.), Feminism and Postmodernism, London, Routledge, 1990. 